\section{Visión Electrónica Más que un estado sólido https://doi.org/10.14483/issn.2248-4728}

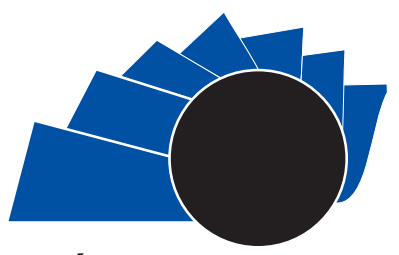

VISIÓN ELECTRONICA

UNIVERSIDAD DISTRITAL

FRANCISCO JOSÉ DE CALDAS

A Research Vision

\title{
Semi-leptonic decays of a particle to three bodies
}

\author{
Decaimiento semi-leptónico de una partícula a tres cuerpos \\ Mauricio Rozo-Clavijo ${ }^{1}$, Carlos Germán Cortés-Hernández ${ }^{2}$, \\ Javier Alberto Olarte-Torres ${ }^{3}$
}

\section{INFORMACIÓN DEL ARTICULO}

Historia del articulo

Enviado: 05/09/2018

Recibido: 19/09/2018

Aceptado: 07/11/2018

\section{Keywords:}

Decay fraction,

Decay width,

Semi-leptonic decay,

Spectator model.

\section{Palabras clave:}

Fracción de decaimiento, Ancho de decaimiento, Decaimiento semi-leptónico, Modelo espectador.

\section{ABSTRACT}

Is presented the dynamics and kinematics of the semi-leptonic decays $D^{+} \rightarrow K^{0} e^{+} v_{e} D^{0} \rightarrow K^{-} \mu^{+} v_{\mu} D^{0} \rightarrow \pi^{-} e^{+} v_{e} \quad \bar{B}^{0} \rightarrow D^{+} e^{-} v_{e} \quad B^{-} \rightarrow D^{0} e^{-} v_{e}$ with interaction $V-A$ calculating the decay width and the fraction of decay for each process using spectator model. The results are compared with the experimental data of the table particle data group, which show that the model prediction for this type of decay can be considered as a first approximation. This type of analysis is particularly important because it allows one hand, introduce to the beginner students to the study of particle physics and on the other gives the possibility to use strategies to address topics modern in physics courses at university level.

\section{RESUMEN:}

Se expone la dinámica y cinemática de los decaimientos semi-leptónicos $D^{+} \rightarrow K^{0} e^{+} v_{e} D^{0} \rightarrow K^{-} \mu^{+} v_{\mu} D^{0} \rightarrow \pi^{-} e^{+} v_{e} \quad \bar{B}^{0} \rightarrow D^{+} e^{-} v_{e} B^{-} \rightarrow D^{0} e^{-} v_{e}$ con interacción $V-A$ calculando el ancho de decaimiento y la fracción de decaimiento para cada proceso haciendo uso del modelo espectador. Los resultados obtenidos son comparados con los datos experimentales de la tabla Particle Data Group, mostrando que la predicción del modelo para este tipo de decaimientos puede ser considerado como una primera aproximación. Este tipo de análisis adquiere especial importancia ya que permite, por un lado, introducir a los estudiantes en el análisis de los decaimientos de partículas y por otro, da la posibilidad de utilizar estrategias para abordar tópicos modernos en cursos de física a nivel universitario.

\footnotetext{
${ }^{1}$ BSc In Physics, Universidad Pedagógica Nacional, Colombia. Specialization in Physical Sciences, Universidad Nacional de Colombia, Colombia. MSc. In Physical Sciences, Universidad Nacional de Colombia, Colombia. Current position: Professor at Universidad Pedagógica Nacional, Colombia. E-mail: mclavijo@pedagogica.edu.co. ORCID: https://orcid.org/0000-0001-6427-8608.

${ }^{2}$ BSc In Physics, Universidad Pedagógica Nacional, Colombia. Current position: Professor at Colegio Parroquial Confraternidad de la Doctrina Cristiana, Colombia. E-mail: matfisast@hotmail.com. ORCID: https://orcid.org/0000-0002-9720-1305.

${ }^{3}$ BSc. In Physics, Universidad estatal de Tbilisi, MSc. and Ph.D. In Physical Sciences, Universidad Nacional de Colombia, Colombia. Current position: Professor at Universidad Distrital Francisco José de Caldas, Colombia. E-mail: jaolartet@udistrital.edu.co. Orcid: https://orcid.org/0000-0002-9173-3685.
} 


\section{Introduction}

Particles sensitive to strong and weak force are called hadrons. The arrangement of quarks for the formation of a hadron can be in two ways; in trios of quarks $\left(q, q^{\prime}, q^{\prime \prime}\right)$ or in quark-antiquark pairs $(\mathrm{q}, \overline{\mathrm{q}})$ The combination of triads of quarks gives rise to heavy particles called baryons. The combination in quark-antiquark pairs $(\mathrm{q}, \overline{\mathrm{q}})$ results in light particles called mesons. The quarks have an intrinsic property called flavor. The different flavor combinations of quarks explain the existence of different kinds of hadrons [1] . In addition, to carry out the different combinations of the quarks-antiquarks it is necessary that they have fractional electric charge of the electric charge of the electron.

The organization of particles for a decay process when you have a set of Nparticles in the final state, is called the decay channel. The decay channel is characterized by the number and nature of particles in which the system can be grouped regardless of the quantum mechanical state of each particle or the state of motion of each particle relative to the others [2].

When you have a system made up of four particles, the organization of the channels is carried out in the following way: $\mathbf{A} \rightarrow \mathbf{a}+\mathbf{b}+\mathbf{c}$ channel $\mathrm{O} ; \mathrm{A} \rightarrow(\mathrm{a}+\mathrm{b})+\mathrm{c}$ channel $1 ; \mathrm{A} \rightarrow \mathrm{a}+(\mathrm{b}+\mathrm{c})$ channel 2 , where the set $(a+b) y / o(b+c)$ form a state [3]. Figure 1 shows the possible forms of grouping of particles for a given decay process,

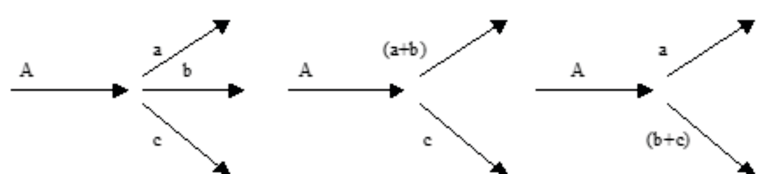

Figure 1. Grouping modes for a process of decay of a particle to three bodies [3].

When you have a decay in which particles $n \geq 4$ are part, being the number of $n$ particles, the number of independent invariant Lorentz variables that characterize the kinematic state of the particles is equal to $3 n-10$ [2]. These variables allow describing the kinematic characteristics of the initial and final state of the particles without considering the dynamic processes that are carried out in the interaction process. The kinematic variables are related to the laws of conservation of energy and momentum and are always valid regardless of the nature of the particles. In this article, an analysis is made on the dynamics and kinematics of the semi-leptonic decay of a particle with three bodies with weak interaction, making use of the spectator model as the first approximation in the explanation to this class of decay.

\section{Spectator model}

One of the first models proposed for the explanation of semi-leptonic decays is the spectator model [4]. In this model, the constituents of the meson are considered, the quarks, linked and non-interacting, in addition, one of them is considered active, which decays independently of his partner as shown in Figure 2

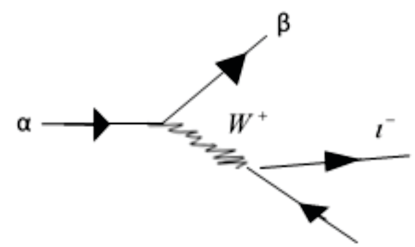

Figure 2. Semi-leptonic decay $\alpha \rightarrow \beta \mathrm{r} \bar{v}_{1}$, being $(\alpha)$ the initial meson $(\beta)$ the outgoing meson $\left(1^{-}\right)$, the loaded lepton $\left(e^{-}, \mu^{-}, \tau^{-}\right), \overline{v_{1}}$ the corresponding antineutrino and $+W$ the intermediate particle [4].

When the mesón $(\alpha)$ change of flavor is emitted mesón $(\beta)$. The flavor change of the active quark of the inn it is due to the action of the weak force that manifests itself through the change of identity, the flavor, of the linked quarks. The quark of the meson incoming transforms into another quark of the meson outgoing $(\beta)$ and the excess energy of the process is carried by the intermediate particle $\left(W^{+}\right)$, that decays in a lepton with its corresponding antineutrino $\left(\bar{\imath} \bar{v}_{1}\right)$. The decaying active quark is considered as being isolated from its antiquark partner. Although strong force is present in the process it cannot change the flavor of quarks [4], [5].

The main characteristic of the model of the spectator quark, is to consider the mesons constituted by a heavy quark of a flavor and with the same order of average life time [4]. Making use of this model, the semi-leptonic decay $D^{0}(c, \bar{u}) \rightarrow \pi^{-}(d, \bar{u}) e^{+} v_{e}$ It can be analyzed as follows: the quark $(c)$ of the meson $(D)$ changes its flavor by transforming into a quark (d) giving rise to meson(p) as illustrated in Figure 3.

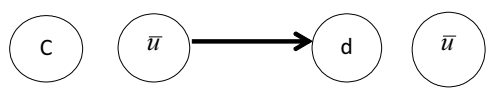

Figure 3. The quark (c) changes flavor to a quark (d). The meson $\left(D^{\circ}\right)$ becomes to meson $\pi$. Source: own. 
When the quark $c$ of the meson $D^{\circ}$ change of flavor is issued meson $\pi$ and the excess energy of the process is carried by the intermediate particle $W^{\text {. }}$. This particle then decays into a lepton with its corresponding neutrino. The predictions of the spectator model for semi-leptonic decay processes with weak interaction can be considered as a first approximation to this class of decays. However, since the viewer model does not consider the linked states in which the quarks are located, this leads to a limitation of the model. Models that consider the linked states of quarks can be obtained in the literature as is the article by Nathan Isgur and Daryl Scora (Semi leptonic B and D decays in the quark model [6], in which the equation of a state is shown bound with a binding potential for strong interaction, obtaining more accurate results with respect to the experimental data.

In the processes of semi-leptonic decay to three bodies, leptons and mesons appear in the final state. In these decays, the energy of the system is distributed equally in the phase space, giving rise to different percentages of decay. In addition, it is important to note that quarks are grouped in pairs giving rise to three classes of families. Transitions between quarks of the same family are more likely to occur than those carried out in different families. Therefore, some semi-leptonic decay processes will have a higher probability of occurrence than others, since transitions between quarks between the same family are more likely than transitions between different families.

The elements of the Cabibbo-Kobayashi-Maskawa matrix (CKM) [7], characterize the transition probability of the quarks between the different families, that is, the transition probability of a quark (q) from one meson, to another quark (q) of another meson is characterized by one of the elements of the matrix ( CKM) based on the electroweak theory framework [8], [9], [10]. The experimental data for each element of the Cabibbo-Kobayashi-Maskawa matrix are [7]:

$V_{i j}=\left(\begin{array}{ccc}V_{u d} & V_{u s} & V_{u b} \\ V_{c d} & V_{c s} & V_{c b} \\ V_{t d} & V_{t s} & V_{t b}\end{array}\right)=\left(\begin{array}{ccc}0.97417 \pm 0.00021 & 0.2248 \pm 0.0006 & (4.09 \pm 0.39) \times 10-3 \\ 0.220 \pm 0.005 & 0.995 \pm 0.016 & (40.5 \pm 1.5) \times 10-3 \\ (8.2 \pm 0.6) \times 10-3 & (40.0 \pm 2.7) \times 10-3 & 1.009 \pm 0.031\end{array}\right)$

The Cabibbo-Kobayashi-Maskawa matrix is also parameterized from the three mixing angles and the phase that violates conjugation-loading (CP) [11], [12], [13]. These parameters are fundamental for the explanation of the processes between particles, so their determination is very important and in general they are obtained considering different semi-leptonic decay processes. The determination of the element $(V u d)$ is carried out from the study of beta decay, however, more precise measurements have been made from the neutron's lifetime [14]. On the other hand, with the experiment PIBETA [15] in which the decay measurement has been improved $(\pi \rightarrow \pi e \mathrm{~V} e)$ you get a better value of the item $\left(V_{u d}\right)$. The determination of the value of $\left(V_{u s}\right)$ is performed by comparing the different semi-leptonic decay modes of the Kaon, obtaining a value of $\left|V_{u d}\right|=0.2237 \pm 0.0009$ [16]. From the semileptonic decays $(D \rightarrow \pi \ell v)$ the value of the element $V_{c d}$ is obtained, $\left|V_{u d}\right|=0.220 \pm 0.005[17][18]$. The determination of the element $(V c s)$ is obtained from semi-leptonic decays $(D \rightarrow K \ell v)$ and leptonic decays obtaining the value of $|V c s|=0.995 \pm 0.016 \quad[17]$. The value of the element $(V c b)$ is obtained by determining the semi-leptonic decays $(B \rightarrow D \ell \bar{v})[19]$, giving a value of $|V c b|=(42.2 \pm 0.8) \times 10^{-3}$. The determination of $\left(V_{u b}\right)$ It is done through semi-leptonic decay $\left(B \rightarrow X_{u, c} \ell \bar{v}\right)$, although it is difficult to measure since there is a great noise in its determination [17], [19]. To find the value of the elements $\left(V_{t d}\right)$ y $\left(V_{t s}\right)$ Its determination is made from oscillations $(B-\bar{B})[17]$. Finally, the measure of the value of the element $\left(V_{t b}\right)$ is done by calculating the decay fraction defined as: $(R=B(t \rightarrow W b) / B(t \rightarrow W q))$ where, $q=b, s, d$ [20], [21].

\section{Decay width and phase space}

The decay width of a given process is defined by two terms that are the Feynman amplitude $(M)$ and the phase space [22]. The Feynman amplitude describes and possesses all the dynamic information of the process, which can be calculated using the Feynman diagrams and rules for the corresponding interaction [22], [23]. The phase space contains all the kinematic information of the process, which depends on the mass, energy and momentum of the particles involved in the interaction [23]. Therefore, the decay width for a given process is defined as [22]:

\section{$d \Gamma \alpha|M|^{2} \cdot($ phase space)}

The decay width of a particle to $\mathrm{N}$ particles can be expressed as follows [22],

$$
\mathrm{d} \Gamma=\frac{1}{2 E_{\alpha}(2 \pi)^{3 n-4}} \sum_{s}|M|^{2} \delta^{4}\left(P_{\alpha}-\sum_{i=1}^{n} P_{i}\right) \prod_{i=1}^{n} \frac{d^{4} p_{i}}{(2 E)_{i}},
$$

where $\left(p_{i}\right)$ is the quadric-moment of the mass particle $\left(m_{i}\right)$ with total energy

$$
E_{i}^{2}-\vec{P}_{i}^{2} c^{2}=m_{i}^{2} c^{4}
$$


The phase space for $(\imath)$ particles in the final state is written in general as [22]:

$$
d_{i}\left(Z \rightarrow a, b, c, \ldots, n_{i}\right)=\delta^{4}\left(P_{Z}-P_{a}-P_{b}-P_{c}, \ldots, P_{n i}\right) \prod_{m=1}^{i} \frac{d^{4} p_{m}}{(2 E)_{m}},
$$

However, when you have many particles in the final state, the phase space can be reduced to subsets of particles where the choice of fractionation depends on the convenience and interest you want to study the process [22], [23]. The Dirac delta function indicates the law of conservation of the energy and momentum of the particles during the interaction.

\section{Decay to three bodies}

A characteristic of weak interactions is the violation of parity [9], which means that we acted only on the left sides of the weak current. This fact leads us to consider the weak currents of a left nature, being that for its description it is convenient to use a combination that specifies this behavior. The way to explain this procedure is to introduce the factor $\gamma_{\mu} \frac{\left(1-\gamma_{s}\right)}{2}$ which establishes a vectorial-axial combination $\left(V^{2}-A\right)$ [4], defined as:

$$
\begin{aligned}
& \sigma_{v}=\gamma_{\mu} \text { Vectorial } \\
& \sigma_{A}=\gamma_{\mu} \gamma_{5} \text { Axial, }
\end{aligned}
$$

and the weak charged current is written as [4], [5], $\quad J_{\mu}=\bar{\psi} \gamma_{\mu}\left(1-\gamma_{5}\right) \psi$.

Consider the decay of a particle to three bodies with interaction $)(A V$-, is to assume that they are of the weak current type. Therefore, the decay width for these processes is generally given by [22]:

$$
\Gamma\left(B \rightarrow Q / v_{l}\right)=|M|^{2} \Phi\left(\mathrm{P}, \mathrm{M}, \mathrm{P}_{\mathrm{i}}, \mathrm{m}_{\mathrm{i}}\right),
$$

where $B, \mathrm{Q}$ are mesons constituted by a pair quarkantiquark $B(b, \bar{b}) ; \mathrm{Q}(\mathrm{q}, \overline{\mathrm{q}})$. The term $I, v_{,}$is the leptonic pair and $\Phi=\Phi\left(\mathrm{P}, \mathrm{M}, \mathrm{P}_{\mathrm{i}}, \mathrm{m}_{\mathrm{i}}\right)$ the phase space.

Using the electroweak model [4], [5] and assuming that the hadron decays independently of the other constituents of meson [4], Feynman's breadth of the process is given by [4], [22]:

$$
M=\frac{G_{f}}{\sqrt{2}} V_{b q} \bar{U}(q) \gamma^{\alpha} \frac{\left(1-\gamma_{5}\right)}{2} U(b) \bar{U}(I) \gamma_{\alpha} \frac{\left(1-\gamma_{5}\right)}{2} v(\bar{v}) .
$$

Taking the square of the absolute value of the Feynman amplitude and replacing the spinorial products by the projection operators $[9],[10],[22]$,

$$
\sum_{s=1,2,3} U(I) \bar{U}(I)=\gamma^{\mu} P_{l \mu}+m_{l}=P_{l}+m_{l}
$$

$$
\begin{aligned}
& \sum_{s=1,2,3} U(q) \bar{U}(q)=\gamma^{\mu} P_{q \mu}+m_{q}=P_{q}+m_{q} \\
& \sum_{s=1,2,3} U(b) \bar{U}(b)=\gamma^{\mu} P_{b \mu}+m_{b}=P_{b}+m_{b}
\end{aligned}
$$

is obtained,

$$
\begin{array}{r}
|M|^{2}=\frac{G^{2} f}{2} V^{2}{ }_{b q} T h\left[\gamma^{\mu}\left(1-\gamma_{5}\right)\left(P_{b}+m_{b} c\right) \gamma^{\mu}\left(1-\gamma_{5}\right)\left(P_{q}+m_{q} c\right)\right] \\
\left.\cdot T r\left\{\gamma_{\mu}\left(1-\gamma_{5}\right)\left(P_{v}+m_{v} c\right) \gamma^{\mu}\left(1-\gamma_{5}\right)\left(P_{1}+m_{1}\right)\right)\right] .
\end{array}
$$

Evaluating the traces of the previous equation, the dynamics of the system is given by:

$$
|M|^{2}=128 G^{2}{ }_{f} V_{b q}^{2}\left(P_{b} \bullet P_{v}\right)\left(P_{q} \bullet P_{l}\right) .
$$

On the other hand, the kinematic contribution of the process is only possible if,

$$
m_{b}>m_{q}+m_{l}+m_{v}
$$

where $m_{b}, m_{q}, m_{l}, m_{v}$ they are the masses of the particles participating in the process. The moments of the final particles $(q)$ y $(l)$ from the reference frame of the particle can be expressed as,

$$
P_{I}=\left[E_{l}^{2}-m_{l}^{2}\right]^{1 / 2} ; P_{q}=\left[E_{q}^{2}-m_{q}^{2}\right]^{1 / 2},
$$

with the respective energies,

$$
E_{l}=\frac{m_{b}{ }^{2}+m_{l}{ }^{2}-m_{q v}{ }^{2}}{2 m_{b}} ; E_{q}=\frac{m_{b}{ }^{2}+m_{q}{ }^{2}-m_{l v}{ }^{2}}{2 m_{b}} .
$$

Therefore, the phase space for the given process is then given by:

$$
d_{3}\left(b \rightarrow q / \bar{v}_{l}\right)=\delta^{4}\left(P_{b}-P_{q}-P_{l}-P_{v}\right) \frac{d^{3} \vec{p}_{q}}{2 E_{q}} \frac{d^{3} \vec{p}_{l}}{2 E_{l}} \frac{d^{3} \vec{p}_{v}}{2 E_{v}} .
$$

Choosing a subset $\mathrm{X}$ formed by the union of the particles $l y l v$, the phase space is given in the form [4],

$$
d_{3}\left(b \rightarrow q / \bar{v}_{l}\right)=\frac{d^{3} \vec{p}_{q}}{2 E_{q}} d_{2}\left(X \rightarrow I \bar{v}_{l}\right)
$$

defining, $\boldsymbol{X}_{\boldsymbol{i}}=\boldsymbol{P}_{\boldsymbol{b}}-\boldsymbol{P}_{\boldsymbol{i}}$ with, $\boldsymbol{i}=\boldsymbol{q}, \boldsymbol{l}$ or $\boldsymbol{v}$. The decay width for the process is then given by:

$$
\alpha \Gamma\left(b \rightarrow q / \bar{v}_{l}\right)=\frac{1}{128 E_{b} \pi^{5}}|M|^{2} \frac{d^{3} \vec{p}_{q}}{2 E_{q}} d_{2}\left(X \rightarrow \mid \bar{v}_{l}\right) .
$$

Replacing the equation (9) in (15), is obtained:

$$
2 E_{q} \frac{d \Gamma}{d^{3} \vec{p}_{q}}=\frac{G^{2}{ }_{f} V^{2}{ }_{b q}}{E_{b} \pi^{5}} \int\left(P_{b} \bullet P_{v}\right)\left(P_{q} \bullet P_{l}\right) d_{2}\left(X \rightarrow I \bar{v}_{l}\right) .
$$

The solution of the integral is [4],

$$
\int\left(P_{b} \bullet P_{v}\right)\left(P_{q} \bullet P_{1}\right) d_{2}\left(X \rightarrow I \bar{v}_{1}\right)=\frac{\pi}{24}\left[g^{\alpha \beta} X_{q}^{2}+2 X_{q}^{\alpha} X_{q}^{\beta}\right] P_{b \alpha} P_{\alpha \beta} .
$$

Calculating the products $P_{b} P_{q}, X_{q} P_{b}$ y $X_{q} P_{q}$, using

$d^{3} \vec{p}_{q}=4 \pi p_{q} E_{q} d E_{q}, x_{q}=\frac{2 E_{q}}{m_{q}}, d E_{q}=\frac{m_{d} d x_{q}}{2}$, and replacing the previous integral in (16), the decay width is:

$$
\frac{a \Gamma}{d x_{q}}=\frac{G^{2},\left|V_{b q}\right|^{2} m_{a} x_{q}}{96 E_{b} \pi^{3}}\left[X_{q}^{2}\left(P_{b}^{2}+P_{q}^{2}\right)+\left(P_{b}^{2}-P_{q}^{2}\right)-2 X_{q}^{4}\right]
$$


Defining, $X_{q}^{2}=m_{b}^{2}\left(1-x_{q}\right)[4]$, is obtained:

$$
\Gamma=\frac{G^{2} f\left|V_{b q}\right|^{2} m_{b}}{96 E_{b} \pi^{3}} \int x_{q}\left[m_{b}^{2}\left(1-x_{q}\right)\left(P_{b}^{2}+P_{q}^{2}\right)+\left(P_{b}^{2}-P_{q}^{2}\right)^{2}-2 m_{b}^{4}\left(1-x_{q}\right)^{2}\right] d x_{q} .
$$

The algebra can be simplified by taking $P_{q}=0$, without losing precision in the result,

$$
\Gamma=\frac{G_{f}^{2}\left|V_{b q}\right|^{2} m_{b}^{6}}{96 E_{b} \pi^{4}} \int\left(3 x_{q}^{2}-2 x_{q}^{3}\right) d x_{q}
$$

The limits of variation of $x_{q}=\frac{2 E_{q}}{m_{b}}$ are:

$$
\frac{2 m_{q}}{m_{b}} \leq \frac{2 E_{q}}{m_{b}} \leq \frac{m_{b}^{2}+m_{q}^{2}}{2 m_{b}^{2}} \text {. }
$$

By replacing the limits of variation in the last integral, the decay width is finally given by:

$$
\Gamma=\frac{G^{2} f\left|V_{b q}\right|^{2} m_{b}^{6}}{96 E_{b} \pi^{4}}\left[\left(\frac{m_{q}{ }^{2}+m_{b}{ }^{2}}{m_{b}^{2}}\right)^{3}-\frac{1}{2}\left(\frac{m_{q}^{2}+m_{b}^{2}}{m_{b}^{2}}\right)^{4}-\left(\frac{2 m_{q}}{m_{b}}\right)^{3}+\left.\frac{1}{2}\left(\frac{2 m_{q}}{m_{b}}\right)^{4}\right|_{j}\right.
$$

The factor $V_{b q}$ that was introduced in (6) gives an account of the transition probability of the quark $b$ of the meson $B$ to quark $q$ of the meson $Q$ in the framework of the electroweak theory [9], [10]. It usually corresponds to an element of the Cabibbo-KobayashiMaskawa matrix which characterizes this probability [7].

\section{Semi-leptonic decays}

Semi-leptonic decays develop

$D^{+} \rightarrow K^{0} e^{+} v_{e}, \quad D^{0} \rightarrow K^{-} \mu^{+} v_{\mu}, \quad D^{0} \rightarrow \pi^{-} e^{+} v_{e}, \quad \bar{B}^{0} \rightarrow D^{+} e^{-} v_{e}, B \rightarrow D^{0} e^{-} v_{e}$

with interaction $V-A$ supported by the spectator model and the previously obtained equations. Table 1 presents the data that will be used to calculate the decay width and the decay fraction of the proposed decays [7].

\begin{tabular}{|l|l|l|}
\hline Name & Simbol & Value \\
\hline Fermi constant & $G_{f}$ & $1.16639 \times 10^{-5} \mathrm{GeV}^{-2}$ \\
\hline Quark mass $c$ & $m_{c}$ & $1.275 \mathrm{GeV}$ \\
\hline Quark mass $s$ & $m_{s}$ & $0.095 \mathrm{GeV}$ \\
\hline Quark mass $d$ & $m_{d}$ & $0.0048 \mathrm{GeV}$ \\
\hline Quark mass $b$ & $m_{b}$ & $4.65 \mathrm{GeV}$ \\
\hline Average life of $D^{+}$ & $\tau_{D^{+}}$ & $1040 \times 10^{-15} \mathrm{~S}$ \\
\hline Average life of $D^{0}$ & $\tau_{D^{0}}$ & $410.1 \times 10^{-15} \mathrm{~S}$ \\
\hline Average life of $\bar{B}^{0}$ & $\tau_{B^{0}}$ & $1.519 \times 10^{-12} \mathrm{~S}$ \\
\hline
\end{tabular}

Table 1. Experimental data used for the decay processes that are calculated. Source: own.

\subsection{Decay $D^{+} \rightarrow \bar{K}^{0} e^{+} v_{e}$}

For the decay to take place $D^{+}(c, d) \rightarrow K^{0}(s, d) e^{+} v_{e}$ the quark cof meson change flavor to a quark $s$ issuing meson $K_{0}$. Figure 4 shows the semi-leptonic decay decay of the meson $D^{+}$:

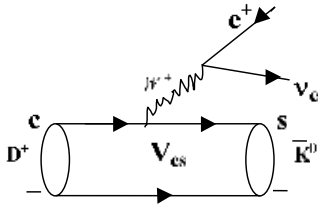

Figure 4. The semi-leptonic decay is shown $D^{+} \rightarrow K^{0} e^{+} v_{e}$ The constant $\left|V_{c s}\right|$ it is a parametrization element of the Cabibbo-Kobayashi-Maskawa matrix. [4] The decay width given by (22) is:

$\Gamma=\frac{G^{2} f\left|V_{c s}\right|^{2} m_{c}^{5}}{96 \pi^{4}}\left[\left(\frac{m_{s}^{2}+m_{c}^{2}}{m_{c}{ }^{2}}\right)^{3}-\frac{1}{2}\left(\frac{m_{s}^{2}+m_{c}^{2}}{m_{c}^{2}}\right)^{4}-\left(\frac{2 m_{s}}{m_{c}}\right)^{3}+\left.\frac{1}{2}\left(\frac{2 m_{s}}{m_{c}}\right)^{4}\right|_{j} ^{7}\right.$,

replacing the corresponding values in Table 1 , we obtain:

$$
\Gamma\left(c \rightarrow \operatorname{se}^{+} v_{e}\right)=2,463168997 \times 10^{-14}\left|V_{c s}\right|^{2} \mathrm{GeV},
$$

or in terms of seconds $\mathrm{s}^{-1}$,

$$
\Gamma\left(c \rightarrow \operatorname{se}^{+} v_{e}\right)=3,744016876 \times 10^{10}\left|V_{c s}\right|^{2} s^{-1} .
$$

The total decay width of the particle is,

$$
\Gamma_{\text {total }}=\frac{1}{\tau}=\frac{1}{1040 \times 10^{-15} \mathrm{~s}}=9,615384615 \times 10^{11} \mathrm{~s}^{-1},
$$

and the fraction of decay is,

$$
\text { fraction of decay }=\frac{\Gamma\left(c \rightarrow s e^{+} v_{e}\right)}{\Gamma_{\text {total }}}=0,039 \cdot\left|V_{c s}\right|^{2} \% .
$$

\subsection{Decay $D^{0} \rightarrow K^{-} \mu^{+} v_{\mu}$}

For the decay to take place $D^{0}(c, u) \rightarrow K^{-}(s, u) \mu^{+} v_{\mu}$ the quark cof meson $D^{\circ}$ must change flavor to a quark $s$ issuing meson $K$. Figure 5 shows the semi-leptonic decay of the meson $D^{\circ}$ :

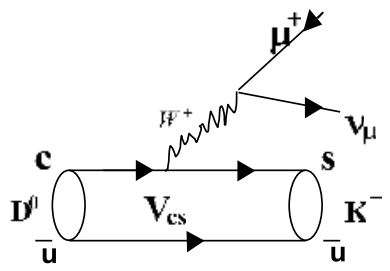

F i g u r e 5. S e m i - l e p t o n i c d e c a y $D^{0} \rightarrow K^{-} \mu^{+} v_{\mu}$. The circles indicate the linked states that make up the mesons $D^{0}, K^{\circ}$. The constant $V_{c s}$ it is an element of the Cabibbo-Kobayashi-Maskawa matrix. [4]

The decay width is:

$$
\Gamma\left(c \rightarrow s \mu^{+} v_{\mu}\right)=2,463168997 \times 10^{-14}\left|V_{c s}\right|^{2} \mathrm{GeV},
$$

or in terms of seconds ${ }^{-1}$,

$$
\Gamma\left(c \rightarrow s \mu^{+} v_{\mu}\right)=3,744016876 \times 10^{10}\left|V_{c s}\right|^{2} s^{-1}
$$

The total decay width of the particle is given by:

$\Gamma_{\text {total }}=\frac{1}{\tau}=\frac{1}{410.1 \times 10^{-15} \mathrm{~s}}=2,438429651 \times 10^{12} \mathrm{~s}^{-1}$. 
and the fraction of decay is,

$$
\text { fraction of decay }=\frac{\Gamma\left(c \rightarrow s \mu^{+} v_{\mu}\right)}{\Gamma_{\text {total }}}=0,015 \cdot\left|V_{c s}\right|^{2} \% \text {. }
$$

\subsection{Decay $D^{0} \rightarrow \pi^{-} e^{+} v_{e}$}

For the decay to take place $D^{0}(c, u) \rightarrow \pi^{-}(d, u) e^{+} v_{e}$, the quark $c$ of meson $D^{0}$ must change flavor to a quark $d$ issuing meson $\pi^{-}$. Figure 6 shows the semi-leptonic decay of the meson $D^{0}$ :

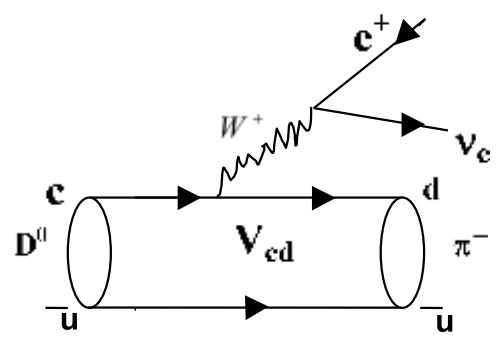

Figure 6. Semi-leptonic decay $D^{0} \rightarrow \pi^{-} e^{+} \nu_{e}$. The constant $c d V$ it is a parametrization element of the Cabibbo-Kobayashi-Maskawa matrix. [4]

You take the fact that $P_{q} \neq 0$ in (22). Therefore, the decay width for this case is given by,

$$
\Gamma\left(c \rightarrow d e^{+} v_{e}\right)=7,699938975 \times 10^{-14}\left|V_{c d}\right|^{2} \mathrm{GeV}
$$

or in terms of seconds ${ }^{-1}$,

$$
\Gamma\left(c \rightarrow d^{+} v_{e}\right)=1,170390724 \times 10^{11}\left|V_{c d}\right|^{2} s^{-1}
$$

The total decay width of the particle is given by:

$\Gamma_{\text {total }}=\frac{1}{\tau}=\frac{1}{410.1 \times 10^{-15} \mathrm{~s}}=2,438429651 \times 10^{12} \mathrm{~s}^{-1}$.

and the fraction of decay is,

$$
\text { fraction of decay }=\frac{\Gamma\left(c \rightarrow d e^{+} v_{e}\right)}{\Gamma_{\text {total }}}=0,048 \cdot\left|V_{c d}\right|^{2} \% .
$$

\subsection{Decay $\bar{B}^{0} \rightarrow D^{+} e^{-} v_{e}$}

For the decay to take place $\bar{B}^{0}(b, \bar{d}) \rightarrow D^{+}(c, \bar{d}) e^{-} v_{e}$, the quark $b$ of meson $\bar{B}^{0}$ must change flavor to a quark $c$ issuing meson $D$. Figure 7 shows the semi-leptonic decay of the meson $\bar{B}^{0}$ :

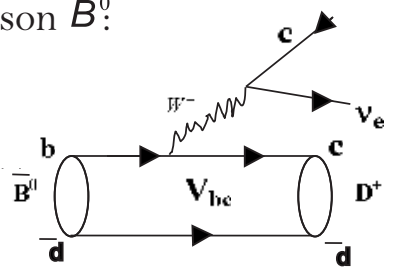

Figure 7. Semi-leptonic decay is indicated $\bar{B}^{0} \rightarrow D^{+} e^{-} v_{e}$. The element $V_{b c}$ it is a parametrization constant of the Cabibbo-Kobayashi-Maskawa matrix. [4]
The decay width is:

$\Gamma\left(b \rightarrow c e^{-} v_{e}\right)=8,064943488 \times 10^{-12}\left|V_{c b}\right|^{2} \mathrm{GeV}$,

or in terms of seconds ${ }^{-1}$,

$\Gamma\left(b \rightarrow c e^{-} v_{e}\right)=1,22587141 \times 10^{13}\left|V_{c b}\right|^{2} s^{-1}$

The total decay width of the particle is given by:

$$
\Gamma_{\text {total }}=\frac{1}{\tau}=\frac{1}{1,519 \times 10^{-12} \mathrm{~s}}=6,583278473 \times 10^{11} \mathrm{~s}^{-1} .
$$

and the fraction of decay is,

$$
\text { fraction of decay }=\frac{\Gamma\left(b \rightarrow c e^{-} v_{e}\right)}{\Gamma_{\text {total }}}=18,62 \cdot\left|V_{c b}\right|^{2} \% .
$$

\subsection{Decay}

$$
B^{-} \rightarrow D^{0} e^{-} v_{e}
$$

For the decay to take place $B^{-}(b, u) \rightarrow D^{0}(c, u) e^{-} v_{e}$, the quark $b$ of meson $B^{-}$must change flavor to a quark $c$ issuing meson $D^{0}$. Figure 7 shows the semi-leptonic decay of the meson $B^{-}$:

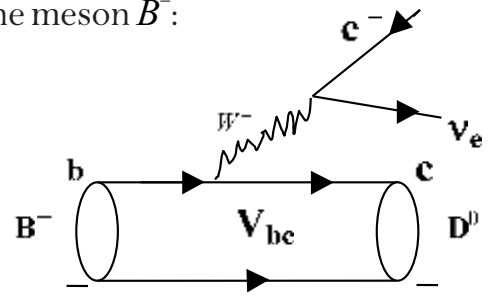

Figure 8. Semi-leptonic decay $B^{-} \rightarrow D^{0} e^{-} v_{e}$. The circles indicate the linked states of the respective meson $B^{-}$y $D^{\circ}$. The constant $\left|V_{c b}\right|$ it is a parametrization element of the Cabibbo-Kobayashi-Maskawa matrix. [4]

The decay width is:

$$
\Gamma\left(b \rightarrow c e^{-} v_{e}\right)=8,064943488 \times 10^{-12}\left|V_{c b}\right|^{2} \mathrm{GeV},
$$

or in terms of seconds ${ }^{-1}$,

$$
\Gamma\left(b \rightarrow c e^{-} v_{e}\right)=1,22587141 \times 10^{13}\left|V_{c b}\right|^{2} s^{-1}
$$

The total decay width of the particle is given by:

$$
\Gamma_{\text {total }}=\frac{1}{\tau}=\frac{1}{1,641 \times 10^{-12} \mathrm{~s}}=6,093845216 \times 10^{11} \mathrm{~s}^{-1} .
$$

and the fraction of decay is,

$$
\text { fraction of decay }=\frac{\Gamma\left(b \rightarrow c e^{-} v_{e}\right)}{\Gamma_{\text {total }}}=20,11 \cdot\left|V_{c b}\right|^{2} \% .
$$




\section{Results}

Table 2 presents the theoretical calculations of the decays using the spectator model, which are compared with the experimental data obtained from the table Particle Data Group [7]

\begin{tabular}{|l|l|l|}
\hline Decay Mode & Spectator Model (\%) & Experimental Data (\%) \\
\hline$D^{+} \rightarrow \bar{K}^{0} e^{+} v_{e}$ & $0.039 \cdot\left|V_{c s}\right|^{2}$ & $8.83 \pm 0.22$ \\
\hline$D^{0} \rightarrow K^{-} \mu^{+} v_{\mu}$ & $0.015 \cdot\left|V_{c s}\right|^{2}$ & $3.30 \pm 0.13$ \\
\hline$D^{0} \rightarrow \pi^{-} e^{+} v_{e}$ & $0.048 \cdot\left|V_{c d}\right|^{2}$ & $0.287 \pm 0.008$ \\
\hline $\bar{B}^{0} \rightarrow D^{+} e^{-} v_{e}$ & $18,62 \cdot\left|V_{c b}\right|^{2}$ & $0.0218 \pm 0.0012$ \\
\hline$B^{-} \rightarrow D^{0} e^{-} v_{e}$ & $20,11 \cdot\left|V_{c b}\right|^{2}$ & $0.0229 \pm 0.0008$ \\
\hline
\end{tabular}

Table 2. The table indicates the fractions of decays calculated using the spectator model. The obtained data are compared with the experimental data. Source: own.

Introducing the values $\left|V_{b q}\right|^{2}$ of the elements of the Cabibbo-Kobayashi-Maskawa matrix [7] involved in each decay process, Table 3 is obtained.

\begin{tabular}{|l|l|}
\hline Spectator Model (\%) & Experimental Data (\%) \\
\hline 0.037 & $8.83 \pm 0.22$ \\
\hline 0.014 & $3.30 \pm 0.13$ \\
\hline $2.434 \times 10^{-3}$ & $0.287 \pm 0.008$ \\
\hline 0.031 & $0.021 \pm 0.0012$ \\
\hline 0.034 & $0.022 \pm 0.0008$ \\
\hline
\end{tabular}

Table 3. A comparison is made between the spectator model and the experimental data given by the Particle Data Group table for the calculated decay processes. Source: own.

On the other hand, the processes of decay $\quad D^{0} \rightarrow K^{0} e^{0} v_{e}, D^{0} \rightarrow K^{\mu} \mu^{v} v_{\mu}$

they require the same taste change $c \rightarrow$ sindicating that the decay width predicted by the spectator model is the same for the particles $D^{0}, D^{+}$. However, when the decay fraction is calculated for both processes, it differs because the half-lives of the particles are different. In

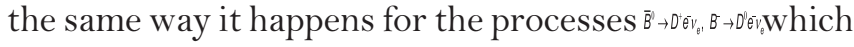
require the taste change $b \rightarrow c$ with the same decay width but with a different decay fraction.

It is important to note that quarks are grouped in pairs giving rise to three classes of families. It can be seen from the elements of the Cabibbo-Kobayashi-Maskawa matrix [7], that transitions between quarks $q \rightarrow q$ ' of the same family are more likely to occur than those carried out in different families. Therefore, the processes of decay $D^{+} \rightarrow K^{0} e^{+} v_{e}, D^{0} \rightarrow K^{-} \mu^{+} v_{\mu}$ where the constant $\left|V_{c s}\right| \approx 1$, are more likely to occur than decay processes $D^{0} \rightarrow \pi^{-} e^{+} v_{e}, \bar{B}^{0} \rightarrow D^{+} e^{-} v_{e}, B^{-} \rightarrow D^{0} e^{-} v_{e}$ where the constants $\left|V_{c d}\right|<1$ y $\left|V_{c b}\right|<1$, since for the last transitions the quarks must decay in different families.

\section{Acknowledgements}

This paper has been made with the support of the Universidad Pedagógica Nacional through the project DFI-307-12 of research Center CIUP.

\section{References}

[1] P. Pascual, "Partículas Elementales Quarks, leptones y unificación de las fuerzas", Prensa científica, 1986.

[2] R. Hagedorn and J. D. Jackson, "Relativistic Kinematics: A Guide to Kinematic Problems of High Energy Physics”, New York: W.A. Benjamin, 1964.

[3] V. I. Gol'danskii and V. V. Kuz'min, "Spontaneous breaking of mirror symmetry in nature and the origin of life", Soviet Physics Uspekhi, vol. 32, no. 1, 1989, pp. 1-29.

[4] R. Phillips and V. Barger, "Collider Physics", USA: Addison-Wesley, 1987.

[5] C. Quigg, "Gauge Theories of the Strong, Weak, and Electromagnetic Interactions", Canada: Addison-Wesley, 1993.

[6] N. Isgur, D. Scora, B. Grinstein and M. Wise, "Semileptonic B and D Decays in the Quark Model”, Physical Review D, vol. 39, no. 3, 1989. https://doi.org/10.1103/PhysRevD.39.799.

[7] C. Patrignani, "Review of Particle Physics", Chinese Physics C, vol. 40, no. 10, 2016.

[8] D. Griffiths, "Introduction to Elementary Particles”, USA: J. W. \& Sons, 1987.

[9] J. D. Bjorken and S. Drell, "Relativistic Quantum Fields”, USA: McGraw-Hill, 1965.

[10] C. Itzykson and J. B. Zuber, "Quantum Field 
Theory”, USA: McGraw-Hill, 1985.

[11] N. Cabibbo, "Unitary Symmetry and Leptonic Decays", Physical Review Letter, vo 1. $10, \quad$ no. $12, \quad 1963$. https://doi.org/10.1103/PhysRevLett.10.531

[12] M. Kobayashi and T. Maskawa, "CPViolation in the Renormalizable Theory of Weak Interaction", Progress of Theoretical Physics, vol. 49, no. 2, 1973, pp. 652-657. https://doi.org/10.1143/PTP.49.652.

[13] L. L. Chau and W. Y. Keung, "Comments on the Parametrization of the KobayashiMaskawa Matrix", Physical Review Letter, vo 1. $53, \quad$ no. $19, \quad 1984$. https://doi.org/10.1103/PhysRevLett.53.180 2.

[14] J. C. Hardy and I. S. Towner, "Superallowed $0+0+$ nuclear $\beta$ decays: 2014 critical survey, with precise results for $\mathrm{V}_{\text {ud }}$ and CKM unitarity", Physical Review C, vol. 91, $\mathrm{n} \mathrm{O} .2 \quad 2, \quad 20015$. https://doi.org/10.1103/PhysRevC.91.02550 $\underline{1}$.

[15] D. Pocanic et al., "Precise Measurement of the Pi+ -> Pio e+ nu Branching Ratio", Physical Review Letter, vol. 93, 2004. https://doi.org/10.1103/PhysRevLett.93.181 $\underline{803}$.

[16] E. Blucher and W. J. Marciano, " $u d V$,us $V$ the Cabibbo Angle and CKM Unitarity”, Chin. Phys., 2014.

[17] S. Aoki et al., "Review of lattice results concerning low energy particle physics," Eur. Phys. J., 2016. https://doi.org/10.1140/epjc/s10052-0164509-7.

[18] L. Widhalm et al." Measurement of Do -> pilnu (Klnu) Form Factors and Absolute Branching Fractions", Physical Review
Let ter, vol. $\quad 97, \quad 2006$. https://doi.org/10.1103/PhysRevLett.97.061 804.

[19] R. Kowalewski and T. Mannel, "Determination of ${ }_{c b} V$ and ${ }_{u b} V$ ", Review of Particle Physics, 2008, pp. 951-965.

[20] D. Acosta et al., "Measurement of $\mathrm{B}(\mathrm{t} \mathrm{Wb}) / \mathrm{B}(\mathrm{t} \mathrm{Wq})$ at the Collider Detector at Fermilab", Physical Review Letters, vol. 95, no. $10,2005$. https://doi.org/10.1103/PhysRevLett.95.102 $\underline{002}$.

[21] V.M. Abazov et al., "Precision Measurement of the Ratio $\mathrm{B}(\mathrm{t} \mathrm{Wb}) / \mathrm{B}(\mathrm{t} \mathrm{Wq})$ and Extraction of $\mathrm{V}_{\mathrm{t}}$, Physical Review Letters, vo 1.107, no. 12,2011 . https://doi.org/10.1103/PhysRevLett.107.12 1802 .

[22] R. P. Feynman, "The Theory of Fundamental Processes", USA: W. A. Benjamin, 1962.

[23] R. Hagedorn, "Relativistic Kinematics", USA: W. A. Benjamin, 1964. 